\title{
Low power transmitter for capsule endoscope
}

\begin{abstract}
This chapter presents the design technique of low power transmitter for the medical application of capsule endoscope. Considering the loss against frequency in a body wireless communication, ISM band of $434 \mathrm{MHz}$ is employed in the design of the transmitter. This band has lower loss and relatively higher data rate compared to other standards. Inductorless architecture was adopted in the circuit design to reduce the circuit area, thus contribute to the reduction of capsule size.The core component of transmitter, the up-conversion mixer and ring oscillator is designed using CMOS $0.13 \mu \mathrm{m}$ technology with voltage supply of $1.2 \mathrm{~V}$. Both the mixer and ring oscillator consumes $1.57 \mathrm{~mA}$ of current, brings the dc power consumption of the transmitter to be $1.88 \mathrm{~mW}$. Data rate of $3.5 \mathrm{Mbps}$ ensure it can transmit high quality medical imaging. The proposed up-conversion and ring oscillator achieved low power and less area while still having the good performance. This achievement makes circuit integration for low power transmitter realizable.
\end{abstract}

Keyword: Transmitter; Capsule endoscope 